\title{
REFLEXIVE MODULES OVER GORENSTEIN RINGS
}

\author{
WOLMER V. VASCONCELOS ${ }^{1}$
}

Introduction. The aim of this paper is to show the relevance of a class of commutative noetherian rings to the study of reflexive modules. They include integrally closed domains, group algebras over these, and Gorenstein rings. We will be basically concerned with a ring $R$ having the following property: Let $M$ be a finitely generated $R$-module; then $M$ is reflexive :f and only if every $R$-sequence of at most two elements is also an $M$-sequence.

In the rest of this note, using the above characterization, we examine the closeness between free modules and Macaulay modules of maximum dimension in a local Gorenstein ring. Finally, it is proved that over a one-dimensional Gorenstein ring only projective modules have projective endomorphism rings.

1. Reflexive modules. As a running hypothesis we will assume that all rings considered here are commutative, noetherian and that unspecified modules are finitely generated. Let $R$ be such a ring and $M$ an $R$-module. Write $M^{*}$ for the group $\operatorname{Hom}_{R}(M, R)$ endowed with the usual $R$-module structure. There is a natural homomorphism $j: M \rightarrow M^{* *}$ and, after [5], $M$ is said to be torsion-less if $j$ is a monomorphism, reflexive if $j$ is an isomorphism. We will also say that $M$ is torsion-free to mean that nonzero elements of $M$ are not annihilated by nonzero divisors of $R$. In other words, if $K$ denotes the full ring of quotients of $R, M$ is torsion-free if and only if the natural homomorphism $M \rightarrow M \otimes K$ is a monomorphism (see Appendix for relation between "torsion-less" and "torsion-free"). Here we want to describe the reflexive modules over a class of rings sensibly vaster than integrally closed domains, but first we recall some notions from [4].

Let $R$ be a local ring. $R$ is said to be a Gorenstein ring if it is CohenMacaulay [9] and whenever $x_{1}, \cdots, x_{n}$ is a maximal $R$-sequence the ideal $\left(x_{1}, \cdots, x_{n}\right)$ is irreducible, i.e., it is not an intersection of properly larger ideals. Equivalently, $R$ is Gorenstein if as an $R$-module $R$ has finite injective dimension. For Krull dimension zero, i.e., for artinian rings, this is the same as having all modules (finitely generated!) reflexive and for Krull dimension one, that torsion-less modules are reflexive. Thus, at least at these low dimensions the Gorenstein property and reflexivity are closely related.

Received by the editors August 7, 1967.

${ }^{1}$ This research was supported by an ONR Research Associateship at Cornell University. 
We use some definitions and notation from [2] and [8]. Let $R$ be a noetherian local ring and let $M$ be a finitely generated $R$-module. An $M$-sequence is a sequence $x_{1}, \cdots, x_{n}$ of elements in the maximal ideal of $R$ such that, if $M \neq 0, x_{i}$ is not a zero divisor of $M /\left(x_{1}, \cdots, x_{i-1}\right) M$ for $i \leqq n$. If $M \neq 0$, the largest integer $n$ for which this happens is called the depth of $M$ or, in symbols, Depth $M$. If $M=0$ we write Depth $M=\infty$. [2] will be used as a reference for the basic properties of Depth, which is there called Codimension.

Definition (1.1). Let $R$ be a commutative noetherian ring, let $M$ be a finitely generated $R$-module and let $k$ be an integer. $M$ is said to have property $S_{k}$ if for every prime ideal $P, \operatorname{Depth} M_{P} \geqq \inf \left(k, \operatorname{dim} R_{P}\right)$.

Definition $(1.1)^{\prime}$. Let $R$ be a commutative ring and let $M$ be an arbitrary $R$-module. $M$ is said to satisfy $S_{k}^{\prime}$ if every $R$-sequence $x_{1}, \cdots, x_{n}$, with $n \leqq k$, is also an $M$-sequence.

For Cohen-Macaulay rings it is proved in [8] that the two definitions coincide.

We now come to the basic notion in this paper:

Definition (1.2). Let $R$ be a commutative noetherian ring. $R$ is said to be quasi-normal if it has property $S_{2}$ and for every prime ideal $P$ of height at most one $R_{P}$ is a Gorenstein ring.

The following are easy consequences of this definition: If $R$ is quasi-normal, so are $R[x]=$ polynomial ring over $R$ and $R[G]=$ group algebra of the finite abelian group $G$. Possibly the same is true for the power series ring $R[[x]]$.

Before characterizing reflexive modules over quasi-normal rings we quote [3, Proposition 4.7]:

Proposition (1.3). Let $N$ be an R-module satisfying $S_{2}^{\prime}$. Then for any $R$-module $M, \operatorname{Hom}(M, N)$ also satisfies $S_{2}^{\prime}$.

Reflexive modules, being duals, enjoy property $S_{2}^{\prime}$. Over quasinormal rings we claim this to be characteristic.

THEOREM (1.4). Let $R$ be a quasi-normal ring and $M$ a finitely generated $R$-module. A necessary and sufficient condition for $M$ to be reflexive is that every $R$-sequence of two or less elements be also an $M$-sequence.

Proof. Let $M$ be a finitely generated $R$-module satisfying $S_{2}^{\prime}$. From the definition of $R$ it is clear that $R$ has no embedded primes. Let $K$ be its total ring of quotients. Since the localization of $K$ at a prime ideal is isomorphic to the localization of $R$ at the corresponding minimal prime, $K$ is a zero-dimensional Gorenstein ring. Consider the exact sequence 


$$
0 \rightarrow M^{\prime} \rightarrow M \stackrel{j}{\rightarrow} M^{* *} \rightarrow M^{\prime \prime} \rightarrow 0
$$

where $j$ is the natural map. By tensoring with $K$ we get $M^{\prime} \otimes K=0$ since $K$ is Gorenstein and so, as remarked earlier, every $K$-module is reflexive. This implies that some nonzero divisor of $R$ annihilates the submodule $M^{\prime}$ of $M$, which is a contradiction, unless $M^{\prime}=0$. Thus $M$ is a torsion-less module. Now localize at any height one prime $P$. Since by hypothesis $R_{P}$ is Gorenstein, torsion-less are reflexive and so $M_{P}^{\prime \prime}=0$. This means that the only associated primes of $M^{\prime \prime}$ have height at least two. Let $P$ be one of them. We get the exact sequence

$$
\begin{aligned}
0 & \rightarrow \operatorname{Hom}(R / P, M) \rightarrow \operatorname{Hom}\left(R / P, M^{* *}\right) \\
& \rightarrow \operatorname{Hom}\left(M / P, M^{\prime \prime}\right) \rightarrow \operatorname{Ext}(R / P, M) .
\end{aligned}
$$

Here $\operatorname{Hom}(R / P, M)=\operatorname{Hom}\left(R / P, M^{* *}\right)=0$ for $P$ contains nonzero divisors and $M$ and $M^{* *}$ are torsion-free. Since height $P \geqq 2$, by assumption $P$ contains an $R$-sequence of length two and by standard properties of Depth, $\operatorname{Ext}(R / P, M)=0$. Hence $\operatorname{Hom}\left(M / P, M^{\prime \prime}\right)=0$, a contradiction if $M^{\prime \prime} \neq 0$, i.e., unless $M$ is reflexive. Q.E.D.

Corollary (1.5). If $R$ is quasi-normal and $N$ is a reflexive $R$ module, for any $R$-module $M \operatorname{Hom}_{R}(M, N)$ is also reflexive.

Corollary (1.6). If $R$ is quasi-normal and

$$
0 \rightarrow M^{\prime} \rightarrow M \rightarrow M^{\prime \prime} \rightarrow 0
$$

is an exact sequence with $M^{\prime}$ and $M^{\prime \prime}$ reflexive, then $M$ is also reflexive.

When $R$ is an integral domain the above characterization of reflexive modules can be expressed in still another way (see [8] for the case of an integrally closed domain). For the moment, $R$ does not have to be noetherian. Let $M$ be a not necessarily finitely generated torsion-free $R$-module. Then

Proposition (1.7). The following are equivalent:

(i) Every $R$-sequence of at most two elements is an $M$-sequence.

(ii) $M=\bigcap_{P \in \mathcal{P}} M_{P}$, where $\mathcal{P}$ is the set of all prime ideals associated to principal ideals.

The proof is implicit in [8]. For an example one can take any flat $R$-module. Using (1.7) then, if in (1.4) $R$ is a domain, one could state that a torsion-free $R$-module is reflexive if and only if it is the intersection of its localizations at the height one primes. 
2. Depth and freeness. In this section $R$ is a Gorenstein local ring and $M$ a finitely generated $R$-module. If $M$ is free then, of course, Depth $M=\operatorname{dim} R$. Here it is shown some properties of free modules shared by modules of maximum Depth. Only a few aspects related to reflexivity will be examined and these could be viewed as consequences of $[4, \S 8]$. First we recall that the Depth of an $R$-module $M$ is given by the following (e.g. [1] or [7]):

Proposition (2.1). Let $M \neq 0$ and let $\operatorname{dim} R=d$. Then Depth $M$ $=d-r$, where $r$ is the largest integer for which $\operatorname{Ext}^{r}(M, R) \neq 0$.

Let $P$ be a prime ideal of the ring $R$. Since $R$ has finite self-injective dimension, so does $R_{P}$. We thus have

Corollary (2.2). If Depth $M=\operatorname{dim} R$ and $M_{P} \neq 0$, then Depth $M_{P}$ $=\operatorname{dim} R_{P}$.

Corollary (2.3). If Depth $M=\operatorname{dim} R$, then $M$ is reflexive and Depth $M^{*}=\operatorname{dim} R$.

Proof. By (2.2) Depth $M_{P} \geqq \inf \left(2, \operatorname{dim} R_{P}\right)$ for any prime ideal $P$, i.e. $M$ satisfies $S_{2}$. As it was said earlier, since $R$ is Cohen-Macaulay, $S_{2}=S_{2}^{\prime}$. Now we apply (1.4) for $R$ is quasi-normal. The second statement follows from $[4, \S 8]$.

Remark. An open question here is whether Depth $M=\operatorname{dim} R$ implies that also Depth $\operatorname{Hom}_{R}(M, M)=\operatorname{dim} R$.

3. Gorenstein rings in dimension one. Let $R$ be an integrally closed domain. Then for every nonzero ideal $I, \operatorname{Hom}_{R}(I, I)=\operatorname{End}_{R}(I)$ $=R$. For other rings this is also true provided the ideal $I$ is large enough. For a ring like $Z[G]=$ integral group ring of the abelian group $G$ however, $\operatorname{End}(I)=Z[G]$ if and only if $I$ is an invertible ideal. More generally

Theorem (3.1). Let $R$ be a one-dimensional Gorenstein ring and $M a$ finitely generated $R$-module. Then $\operatorname{End}_{R}(M)$ is a projective module if and only if $M$ itself is projective.

The proof will follow after a few preliminary steps. One can assume that $R$ is a local ring. Let $M \neq 0$; if $\operatorname{End}(M)$ is projective, to show that $M$ is projective it is enough to prove that $M$ contains a summand isomorphic to $R$.

(i) If $\operatorname{Hom}(M, M)$ is $R$-free, it is easy to see that the maximal ideal $m$ of $R$ is not associated to $M$, i.e., $m$ is not the annihilator of a nonzero element of $M[3$, Lemma 4.5$]$. Thus Depth $M=1$. Let $x$ be an 
element of $\boldsymbol{m}$ which is not a zero divisor for either $R$ or $M$. The exact sequence

$$
0 \rightarrow R \stackrel{x}{\rightarrow} R \rightarrow R /(x) \rightarrow 0
$$

leads to

$$
0 \rightarrow \operatorname{Hom}(M, R) \stackrel{x}{\rightarrow} \operatorname{Hom}(M, R) \rightarrow \operatorname{Hom}(M, R /(x)) \rightarrow \operatorname{Ext}^{1}(M, R) .
$$

Since Depth $M=1$, by (2.1) $\operatorname{Ext}^{1}(M, R)=0$ and we have $\operatorname{Hom}_{R^{\prime}}\left(M^{\prime}, R^{\prime}\right)=\operatorname{Hom}(M, R) \otimes R^{\prime}$ with $M^{\prime}=M / x M$ and $R^{\prime}=R /(x)$.

(ii) Consider the trace map $T: M^{*} \otimes M \rightarrow R$ given by $T(f \otimes m)$ $=f(m)$. It is obvious that $M$ contains a summand isomorphic to $R$ if and only if $T$ is an epimorphism ( $R$ is local!). From the preceding step we have the corresponding diagram of trace maps

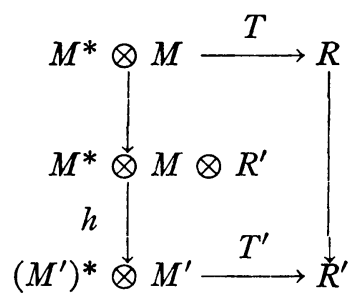

where $h$ is an isomorphism. Thus to show that $T$ is an epimorphism it is enough to show that $T^{\prime}$ is so.

(iii) The exact sequence

$$
0 \rightarrow M \stackrel{x}{\rightarrow} M \rightarrow M^{\prime} \rightarrow 0
$$

leads to the exactness of

$$
0 \rightarrow \operatorname{Hom}(M, M) \stackrel{x}{\rightarrow} \operatorname{Hom}(M, M) \rightarrow \operatorname{Hom}\left(M^{\prime}, M^{\prime}\right)
$$

and we have that $\operatorname{Hom}\left(M^{\prime}, M^{\prime}\right)$ contains a free $R^{\prime}$-module. Thus, in particular, $M^{\prime}$ is $R^{\prime}$-faithful. Now $R^{\prime}$ is a zero-dimensional Gorenstein ring, i.e., $R^{\prime}$ is self-injective. Here this just means that $R^{\prime}$ contains a unique nonzero minimal ideal. From this it follows immediately that every faithful $R^{\prime}$-module contains a direct summand isomorphic to $R^{\prime}$. This completes the proof of the theorem since the converse is trivial.

REMARK. The theorem is not true if $\operatorname{dim} R>1$, even if we assume that $M$ is reflexive. This occurs, for instance, in $R[x, y, z] /\left(x y-z^{2}\right)$, 
where $R$ denotes the real numbers. This ring is normal, Gorenstein but not locally a UFD and so any height one prime which is not invertible gives a counterexample.

\section{ApPENDIX}

We shall clarify here the relationship between torsion-less and torsion-free modules. The same finiteness assumptions of before are still in force. If an $R$-module $M$ is torsion-less, the natural monomorphism $M_{\rightarrow}^{j} M^{* *}$ implies that $M$ can be embedded in a free $R$ module and is thus torsion-free. For the converse

Theorem (A.1). For the commutative noetherian ring $R$, "torsionfree" and "torsion-less" are equivalent notions if and only if $R$ has no embedded primes and $R_{P}$ is a Gorenstein ring for every minimal prime $P$.

Proof. Assume that $R$ satisfies the ring-theoretic statement above and let $M$ be a torsion-free $R$-module. Consider the exact sequence

$$
0 \rightarrow M^{\prime} \rightarrow M \stackrel{j}{\rightarrow} M^{* *}
$$

for which we want to prove $M^{\prime}=0$. If $P$ is a minimal prime, $R_{P}$ is a zero-dimensional Gorenstein ring and so every $R_{P}$-module is reflexive; thus $M_{P}^{\prime}=0$. This says that the annihilator of $M^{\prime}$ is outside of any minimal prime and so contains a nonzero divisor for $R$ has no embedded prime. Since $M^{\prime}$ is a submodule of $M$ this implies $M^{\prime}=0$.

Conversely, assume "torsion-free" = "torsion-less." Let $K$ be the total ring of quotients of $R$ and let $N$ be a finitely generated $K$ module. It is clear that we can find a finitely generated $R$-submodule $M$ of $N$, such that $K \otimes M=K M=N$. Since $M$ is torsion-free by construction, by assumption it is torsion-less. It follows easily that as a $K$-module $N$ is also torsion-less. Now it must be shown that $K$ is a zero-dimensional Gorenstein ring, i.e., that $K$ is self-injective. Let $I$ be an ideal of $K$ which is not an annihilator or in other words, $I \neq \operatorname{Ann}\left(I^{\prime}\right)=$ annihilator of the ideal $I^{\prime}$, for any $I^{\prime}$. Denote $K / I$ by $M$. Then $M^{*}=\operatorname{Hom}(M, K)=\operatorname{Ann}(I)=J$ and $M^{* *}=\operatorname{Hom}(J, K)$. Let $L=\operatorname{Ann}(J) ; I$ is properly contained in $L$. Pick $a \in L-I$, then in $M^{j} \rightarrow M^{* *}, j(a)=0$. Our assumption is then incorrect and thus every ideal is an annihilator. This is however equivalent to $K$ being selfinjective [6].

\section{REFERENCES}

1. M. Auslander, Remarks on a theorem of Bourbaki, Nagoya J. Math. 27 (1966), 361-369. 
2. M. Auslander and D. Buchsbaum, Homological dimension in local rings, Trans. Amer. Math. Soc. 85 (1957), 390-405.

3. M. Auslander and O. Goldman, Maximal orders, Trans. Amer. Math. Soc. 97 (1960), 1-24.

4. H. Bass, On the ubiquity of Gorenstein rings, Math. Z. 82 (1963), 8-28.

5. - Injective dimension in noetherian rings, Trans. Amer. Math. Soc. 102 (1962), 18-29.

6. J. P. Jans, Duality in noetherian rings, Proc. Amer. Math. Soc. 12 (1961), $829-835$.

7. G. Levin and W. V. Vasconcelos, Homological dimensions and Macaulay rings, Pacific J. Math. 25 (1968), 315-323.

8. P. Samuel, Anneaux graduês factoriels et modules réflexifs, Bull. Soc. Math. France 92 (1964), 237-249.

9. O. Zariski and P. Samuel, Commutative algebra, Vol. II, Van Nostrand, Princeton, N. J., 1960.

Cornell University 